\title{
A HUMANIZAÇÃO DOS ESPAÇOS NA REDE SARAH DE HOSPITAIS DE REABILITAÇÃO
}

Flávia Fushimi

Universidade Estadual de Londrina - UEL, Especialização em Projeto Arquitetônico: Composição e Tecnologia do Espaço Construído, Londrina, PR. E-mail: flavia.fushimi@hotmail.com

\section{RESUMO}

O presente trabalho tem por objetivo realizar um breve estudo sobre a questão da humanização dos espaços nos projetos da Rede SARAH de Hospitais de Reabilitação, idealizados pelo arquiteto João Filgueiras Lima (Lelé).Com base nos estudos relacionados à humanização dos espaços assim como da importância da figura do arquiteto perante a elaboração de ambientes hospitalares foi possível realizar uma análise das unidades do SARAH a partir de diversos elementos e atributos utilizados por Lelé com o intuito de influenciar positivamente no processo de cura e na melhora dos pacientes, promover o bem estar e tranquilidade dos mesmos, além de afastar da padronização e instituicionalização predominante nesta área. Dessa forma, por intermédio da arquitetura humanizada em seus projetos, o arquiteto cria um novo conceito de hospitais ao longo de sua produção arquitetônica, adequando os projetos da rede às necessidades tecnológicas e ambientais e proporcionando espaços acolhedores, saudáveis e alegres.

Palavras-chave:Humanização, Rede SARAH, João Filgueiras Lima, Arquitetura Humanizada.

\section{THE HUMANIZATION OF SPACES IN REDE SARAH DE HOSPITAIS DE REABILITAÇÃO}

\section{ABSTRACT}

The present work aims to carry out a brief study about humanization of spaces in Rede SARAH Hospitais de Reabilitação projects, developed by architect João Filgueiras Lima (Lelé). Based on studies related to humanization of spaces, as well as importance of architect figure in elaboration of hospital's ambients was possible to make an analysis at SARAH unities from many elements and attributes used by Lelé with purpose to influence positively in healing process and recovery patients, to promote welfare and tranquility of the same and to repel the standardization and institucionalization prevailing in this area. Therefore, through humanization architecture in your projects, the architect creates a new concept of hospitals over your architectural producing, adjusting the projects to technologies and environmental needs and providing welcoming, healthy and happy spaces.

Keywords: Humanization, Rede SARAH, João Filgueiras Lima, Humanization architecture.

\section{INTRODUÇÃO}

A palavra "humanizar" tem como significado atribuir caráter humano; conceder ou possuir condição humana a qualquer coisa ou lugar. Segundo Vasconcelos (2004), para humanizar é necessário entender o conceito de ser humano bem como ter consciência de que a pessoa que utiliza o espaço é fundamental na definição de como deve ser o ambiente. Dessa forma, a humanização está intimamente ligada aos ambientes da área da saúde, onde a figura do arquiteto perante a elaboração de tais ambientes passa a se tornar imprescindível, especialmente no processo de criação de espaços que estabeleçam uma significativa relação com os usuários, influenciando positivamente no processo de cura.

A mesma preocupação em atender às necessidades dos usuários dentro de um ambiente hospitalar mostra-se presente nos projetos da Rede SARAH de Hospitais de Reabilitação, 
idealizados pelo arquiteto João Filgueiras Lima, conhecido como Lelé. Este cria um novo conceito de hospitais a partir de sua trajetória e sua produção arquitetônica na área hospitalar ao longo dos anos, adequando os projetos da rede às necessidades tecnológicas e ambientais.

Composta por dez unidades em diversas regiões do país, a Rede SARAH de Hospitais de Reabilitação, reconhecida nacional e internacionalmente, atua no atendimento de alta qualidade a todos os níveis da população e em diferentes programas de reabilitação, os quais são definidos em cada unidade conforme os indicadores epidemiológicos de cada região em que cada unidade está localizada.

As unidades que compõem a Rede Sarah são projetos de autoria de Lelé (com exceção da unidade de Belo Horizonte, onde foi realizado o projeto de reforma pelo arquiteto), que buscou no desenvolvimento dos projetos uma caracterização na integração de sua concepção arquitetônica bastante singular e marcante aos diferentes programas de reabilitação propostos para cada unidade. Assim, o trabalho proposto tem por objetivo realizar um breve estudo sobre a questão da humanização dos espaços nos projetos da Rede SARAH de Hospitais de Reabilitação.

\section{METODOLOGIA}

Para atingir o objetivo proposto, foram realizadas pesquisas bibliográficas referentes ao tema, como a humanização dos espaços em projetos arquitetônicos (com ênfase em ambientes da área da saúde), assim como a produção arquitetônica e metodologia projetual de João Filgueiras Lima; levantamento das unidades da Rede SARAH de Hospitais de Reabilitação no país juntamente com suas especificações e características e, por fim, análise projetual das unidades a fim de localizar os diversos atributos chaves presentes no processo de desenvolvimento do arquiteto.

\section{RESULTADOS}

Por meio da sensibilidade na criação dos espaços da Rede SARAH, Lelé implanta um novo padrão na questão da arquitetura hospitalar por intermédio da inserção de diversos atributos chaves no processo de idealização dos projetos da Rede SARAH, tais como: conforto ambiental; relação dos ambientes internos com os externos, e por fim, elementos artísticos na parceira com o artista plástico Athos Bulcão, que serão abordados a seguir:

- Conforto ambiental: em seus projetos Lelé caracteriza-se em atender da melhor forma possível ao conforto ambiental, seja no aproveitamento máximo dos ventos dominantes e da iluminação natural, topografia, clima da região, escala da edificação e da vegetação existente. Nos projetos da Rede SARAH, que são grandes referências da arquitetura bioclimática, Lelé desenvolve uma série de tecnologias e soluções arquitetônicas tornando os edifícios mais confortáveis, saudáveis, econômicos, além de esteticamente agradáveis e, sobretudo humanizados.

Segundo o próprio Lelé ${ }^{1}$, a ideia de priorizar os sistemas naturais em seus trabalhos começou no ambiente hospitalar, já que nestes locais era necessária a humanização dos ambientes. Nos hospitais, a climatização por meio da ventilação natural era uma forma de controlar a infecção hospitalar, já que anterior a década de 70 a sustentabilidade não exercia um papel relevante na composição de um projeto.

\footnotetext{
${ }^{1}$ Em entrevista concedida a Jotabê Medeiros para O Estado de São Paulo em 2008. "Informações extraídas da bibliografia 'A arquitetura de Lelé: fábrica e invenção” de Max Risselada e Giancarlo Latorraca (2010). Colloquium Socialis, Presidente Prudente, v. 01, n. Especial 2, Jul/Dez, 2017, p.624-630. DOI: 10.5747/cs.2017.v01.nesp2.s0203
} 
Figura 1- Croqui da cobertura da espera do ambulatório, Sarah-Belo Horizonte.

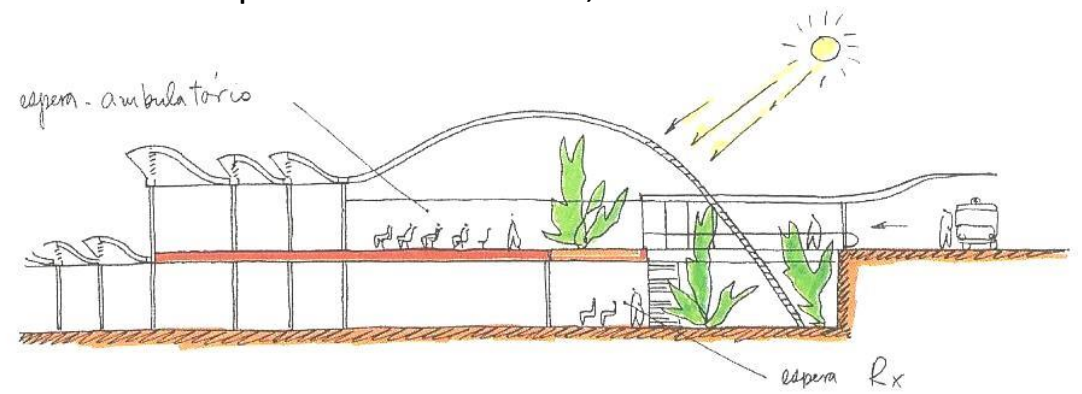

Fonte: Risselada e Latorraca (2010).

Nota-se que ao longo de seus projetos há um aprimoramento no quesito da arquitetura bioclimática, onde as edificações passam a evoluir em vários aspectos, uma vez que o arquiteto participa de todas as etapas de suas produções: desenvolvimento do projeto, execução e manutenção, adotando soluções de acordo com as especificidades de cada projeto proposto.

O quadro 1 demonstra algumas soluções e técnicas utilizadas por Lelé a fim de proporcionar o conforto desejado nos ambientes dos hospitais e centros da rede, levando em considerações as particularidades existentes em cada projeto proposto.

Quadro 1 - Soluções e técnicas utilizadas por Lelé na Rede SARAH.

\section{CONFORTO AMBIENTAL - REDE SARAH}

Brasília - DF

- Adoção de sistemas simples de iluminação e ventilação naturais;

- Uso de sistema de ar condicionado e controle de iluminação artificial em ambientes necessários

(centro curúrgico, esterilização, raio-x etc).
Brasília Lago - Norte

- Inserção de sistema simples de ventilação natural - ar extraído pelas aberturas de sheds semelhantes à Salvador; - Utilização de sequência de plataformas interligadas por taludes e rampas para vencer

0
São Luís - MA

- Abertura de ventilação zenital, construção em meio ao pátio e jardins com pé direito alto, a fim de favorecer o clima da cidade; - Partido horizontal levando em consideração a expansão da edificação.

terreno em declive.

\section{Salvador - BA}

- Sheds com formato simples de teto curvo;

- Duto de distribuição de ar fresco;

- Processo de terraplanagem a fim de adequar o edifício no terreno.
Belo Horizonte - MG

- Cobertura em sheds (dispositivos na cobertura que favorecem iluminação e ventilação natural) semelhantes à Salvador; - Inserção brises intempéries;

- Cobertura em arco no auditório, hall de entrada e espera do ambulatório.
Rio de Janeiro - RJ

- Sheds constituindo unidades onduladas;

- Instalação de ar condicionado mecânico em paralelo com o sistema de ventilação natural; - Introdução de basculantes móveis em ambientes com vidro verticais. 


\section{Fortaleza - CE}

- Implantação de galerias de tubulação com duto de ventilação no pavimento térreo;

- Pulverização de águas no lado externo junto a espelhos d'água;

- Cobertura em formato diferente por conta do clima da região.

\section{Macapá - AP}

- Aumento das aberturas de ventilação zenital dos prédios por conta do clima da região;

- Criação de pés direitos relativamente altos para favorecer extração de ar quente pelas aberturas dos sheds;

- Aumento da velocidade de ventilação interna através de ventiladores específicos.

\section{Belém - PA}

- Inserção de sheds semelhante à unidade de Macapá por conta do clima da região; - Implantação do centro favorecendo os ventos predominantes.

Fonte: Lima (1999), Latorraca (2000) e Risselada e Latorraca (2010), organizado pela autora (2017).

- Relação interior e exterior: a relação dos espaços internos com o externo e o contato com natureza apresenta-se como fatores relevantes na qualidade da humanização dos ambientes hospitalares, já que os estímulos provenientes do ambiente externo provocam diferentes reações, sejam elas sensoriais, auditivas, olfativas e etc.

Essa relação interior/exterior traz benefícios aos pacientes por provocar estímulos em seus sistemas sensoriais, a partir dos elementos ambientais que possui, causando diferentes percepções do ambiente. (VASCONCELOS, 2004, p. 10).

Lelé ressalta em seus projetos do SARAH a questão da relação do usuário com o entorno, possibilitando a integração dos espaços a grandes áreas ajardinadas e ampla visualização da paisagem, além do contato direto com a natureza. Tais ambientes são destinados aos banhos de sol e desenvolvimento de diversas atividades terapêuticas individuais ou em grupo ao ar livre, que contribuem qualitativamente no tratamento (auxiliando no processo de cura), proporcionam bem estar, além de promover socialização, lazer, cultura e arte aos usuários

A relação do usuário com entorno e a integração dos espaços dentro das edificações da Rede SARAH mostra-se presente em todos os projetos de Lelé (Quadro 2). Ainda que existam algumas particularidades em determinadas unidades para tais finalidades, o arquiteto busca soluções a fim de estimular o paciente em seu tratamento bem como proporcionar um ambiente agradável.

Quadro 2- Relação do usuário com o entorno nas unidades.

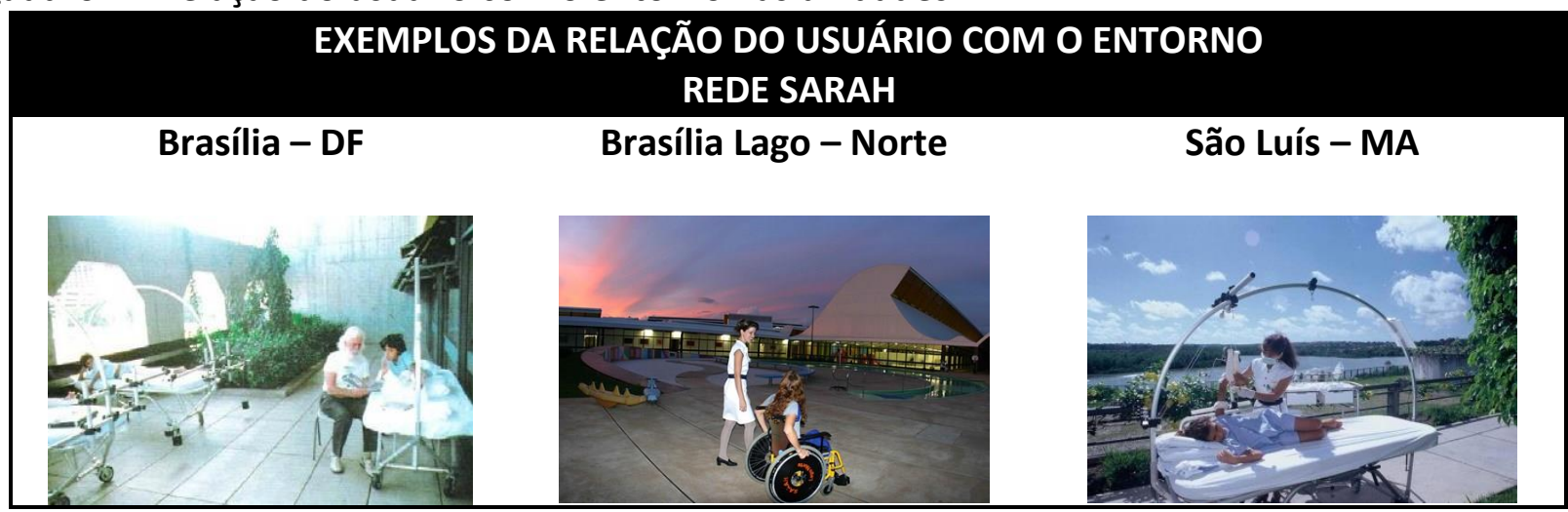

Colloquium Socialis, Presidente Prudente, v. 01, n. Especial 2, Jul/Dez, 2017, p.624-630. DOI: 10.5747/cs.2017.v01.nesp2.s0203 


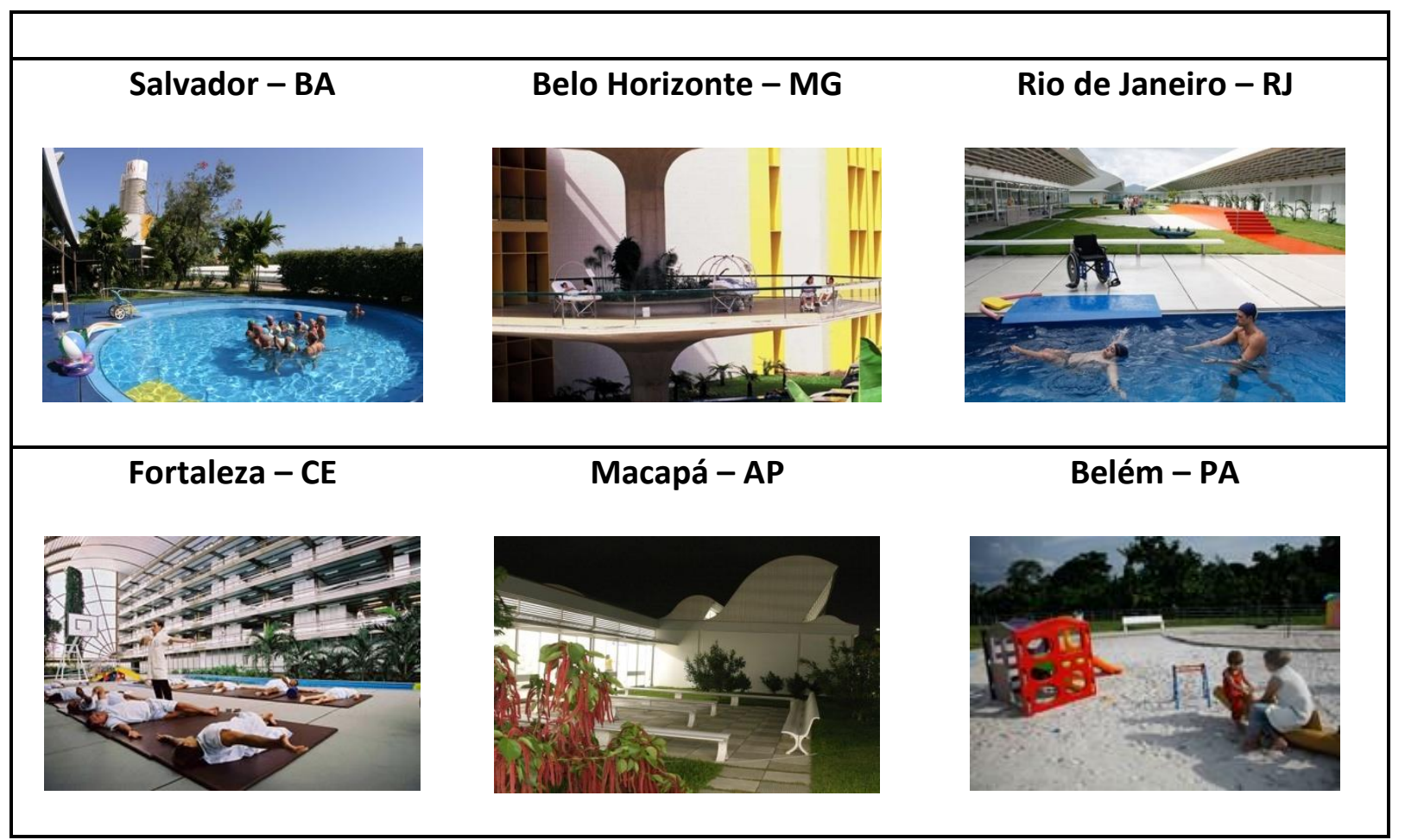

Fonte: Latorraca (2000) e Rede SARAH (2013); organizado pela autora (2017).

- Arquitetura e arte: Lelé trabalha, além da relação do usuário com o entorno e do conforto ambiental das edificações, na questão da integração da arquitetura com a arte para atingir com excelência a humanização em ambientes da área da saúde. Essa união, onde a tecnologia incorpora a arte, resulta na parceria - desde o ano de 1959 - do arquiteto com o artista plástico Athos Bulcão, colaborador de Lelé ao longo de sua carreira.

Athos, que desenvolveu uma série de produções artísticas em todas as unidades, que vão desde fechamentos por painéis até peças desenvolvidas pelo artista para playground, não apenas sugere como desenvolve nos hospitais da rede a questão a utilização de cores vibrantes em suas obras, contrariando a predominância de cores neutras dos hospitais. De acordo com Porto (2010), Lelé sempre discordou da psicologia hospitalar que ditava a predominância de cores neutras para o paciente se acalmar e acreditava que toda emoção positiva que auxilia na cura dos pacientes se torna terapêutica.

Lelé afirma que tanto os painéis como equipamentos criados por Athos Bulcão presentes nos hospitais da rede SARAH (Quadro 3) fazem parte do próprio ambiente, utilizados como uma contribuição integrada à arquitetura do local. Através da humanização destes espaços, o paciente passa a se sentir mais valorizado e respeitado em contato com obras de arte.

Para Lelé, trata-se de uma experiência plástica integrada que contribui ao conjunto de elementos trabalhados para alcançar o conforto e o bem-estar do usuário, considerada na composição do ambiente pretendido como qualquer outro aspecto técnico fundamental para o funcionamento do edifício. (RISSELADA; LATORRACA, 2010, p. 11).

Quadro3- Produções artísticas de Athos Bulcão na Rede SARAH.

\begin{tabular}{|ccc|}
\hline \multicolumn{3}{|c|}{ EXEMPLOS DE PRODUÇÕES ARTÍSTICAS POR ATHOS BULCÃO } \\
REDE SARAH & São Luís - MA \\
\hline Brasília - DF & Brasília Lago - Norte & Sân
\end{tabular}

Colloquium Socialis, Presidente Prudente, v. 01, n. Especial 2, Jul/Dez, 2017, p.624-630. DOI: 10.5747/cs.2017.v01.nesp2.s0203 


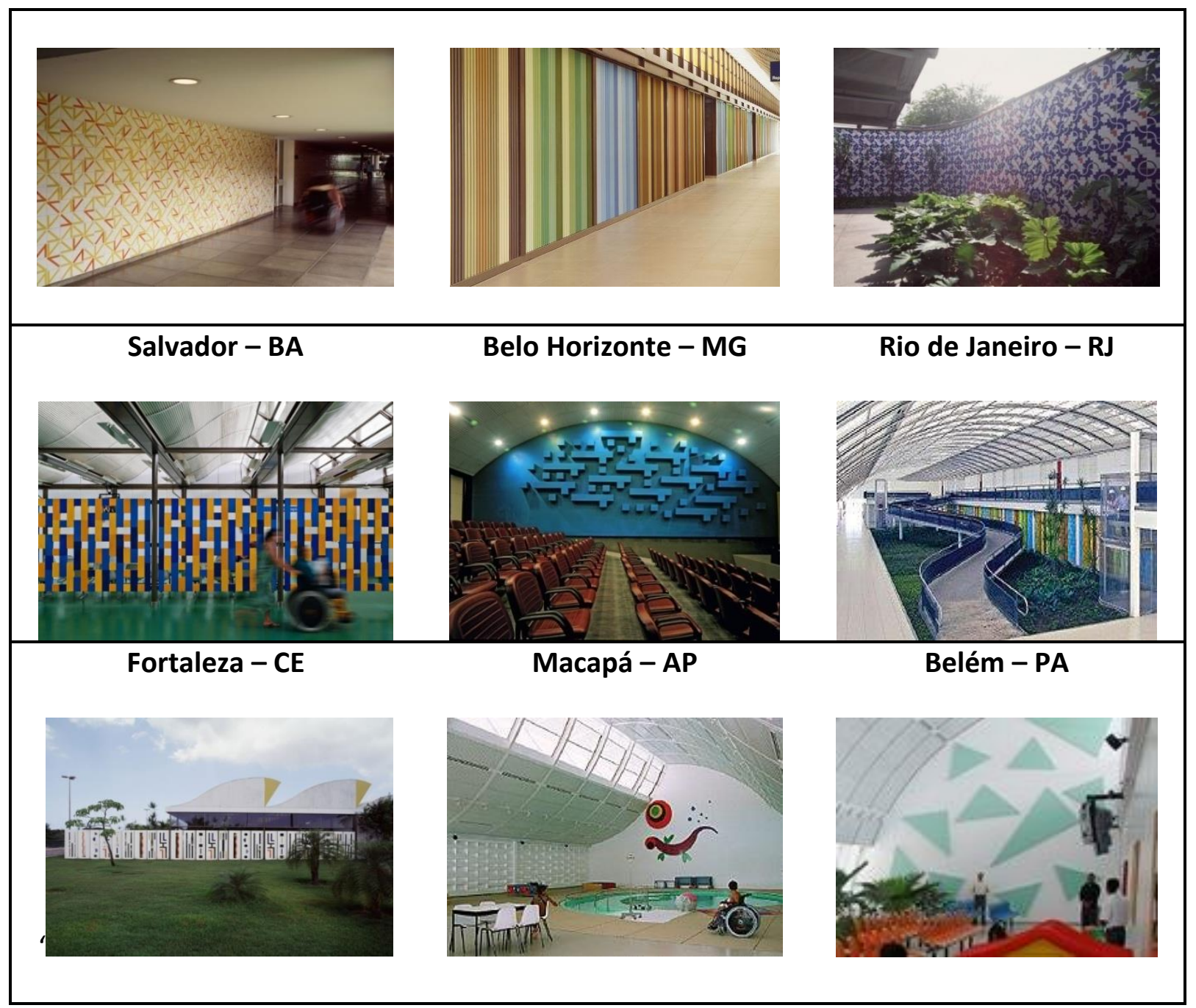

Fonte: Piniweb (2008), Rede SARAH (2013), Fundathos (2014); organizado pela autora (2017).

\section{DISCUSSÃO}

A figura do arquiteto perante a elaboração de ambientes voltados à área da saúde tornarse imprescindível no processo de criação de espaços que estabeleçam uma significativa relação com os usuários, uma vez que influenciam positivamente no processo de cura e na melhora dos pacientes. Segundo o próprio arquiteto:

[...] quando um arquiteto se especializa, ele passa a ver o espaço hospitalar como um espaço tecnológico [...]. Esse espaço começa a carecer de uma função humana, que é a questão primordial do arquiteto. [...] $\mathrm{O}$ fundamental é você criar um espaço em que o paciente se sinta bem, em que realmente esteja psicologicamente preparado para ser curado, se não, não vai ser curado. (LIMA apud RISÉRIO, 2010, p. 42).

Para Aloysio Campos da Paz Júnior (1999), coidealizador da rede e cirurgião chefe da mesma, os hospitais projetados por Lelé expressam o próprio sentido da palavra "reabilitação", pois ao contrário dos espaços constrangedores de sofrimento encontrados em ambientes hospitalares, o arquiteto torna os locais amenos, generosos e ricos em volume e cores, permitindo assim, a humanização do processo de tratamento dos pacientes. 
A parceria entre Lelé e Paz Júnior foi essencial no desenvolvimento dos projetos de Rede SARAH, pois tornaram os hospitais diferenciados através do humanismo e na sensibilidade da criação dos espaços, influenciando intensamente a cultura médica implantada.

CONCLUSÃO

A Rede SARAH de Hospitais de Reabilitação constitui-se em uma das evidências de que edifícios hospitalares, práticas médicas, ambientes humanizados, arquitetura e tecnologia podem, de fato, se atrelar, sendo executadas com excelência e desempenhando papel relevante na assistência médica, ensino, pesquisas e projetos de prevenção relacionados à reabilitação dos indivíduos.

Por meio da sensibilidade na criação dos espaços, Lelé cria um novo conceito de hospitais a partir da sua produção arquitetônica na área hospitalar ao longo dos anos, adequando os projetos da rede às necessidades tecnológicas e ambientais, ao proporcionar espaços acolhedores, saudáveis e alegres, essenciais no processo de tratamento dos pacientes, além de afastar do aspecto hostil, da padronização e da institucionalização predominante nesta área.

A inserção dos diversos atributos utilizados no processo de idealização dos projetos da Rede SARAH que foram abordados no presente trabalho possibilitam a integração da humanização e o enriquecimento dos espaços com a funcionalidade necessária nos hospitais, ou seja, trata-se de um exemplo positivo entre um edifício hospitalar e as práticas médicas que nele se realizam com uma arquitetura pensada para o usuário, tornando um ambiente prazeroso.

\section{REFERÊNCIAS BIBLIOGRÁFICAS}

LATORRACA, G. João Filgueiras Lima, Lelé. São Paulo: Blau, Intituto Lina Bo e P.M, 2000. 263 f.

LIMA, J. F. L. CTRS - Centro de Tecnologia da Rede SARAH. Sarah Letras, 1999. 59 f.

LIMA, J. F. L. Arquitetura. Uma experiência na área da saúde. São Paulo: Romano Guerra, 2012. $336 \mathrm{f}$.

PAZ JÚNIOR, A. C. Lelé. In: LIMA, J. F. L. CTRS - Centro de Tecnologia da Rede SARAH. Sarah Letras, 1999. $59 \mathrm{f}$.

PORTO, C. E. Quando arte e arquitetura se mesclam: a obra de Athos Bulcão e Lelé. In: Agnaldo Farias; Fernanda Fernandes. (Org.). VII Fórum Brasília de Artes Visuais. Arte e Arquitetura: Balanço e Novas Direções. 01 ed. Brasília-DF: Editora UnB, 2010, v. 01, p. 63-80

RISSELADA, M.; LATORRACA, G. A arquitetura de Lelé: fábrica e invenção. São Paulo: Museu da Casa Brasileira, 2010. $244 \mathrm{f}$.

VASCONCELOS, R. T. B. Humanização de ambientes hospitalares: características arquitetônicas responsáveis pela integração interior/exterior. 2004. 176 f. Dissertação (Mestre em Arquitetura e Urbanismo), Universidade Federal de Santa Catarina, Florianópolis, 2004. 